Artículo científico

Volumen 33(1): Artículo 46140, 2022

e-ISSN 2215-3608, doi:10.15517/am.v33i1.46140

https://revistas.ucr.ac.cr/index.php/agromeso/index

\title{
Calidad microbiológica y fisicoquímica y sabor de huevos de gallina de producción convencional o pastoreo ${ }^{1}$
}

\section{Microbiological and physicochemical quality and flavor of hen eggs produced under grazing or conventional systems}

\author{
Marie Guier-Serrano², Gabriela Davidovich-Young², Eric Wong-González², Elba Cubero-Castillo²
}

1 Recepción: 23 de marzo, 2021. Aceptación: 9 de agosto, 2021. Este proyecto fue parte de la tesis de licenciatura de la primera autora, llevada a cabo en la Escuela de Tecnología de Alimentos de la Universidad de Costa Rica, así como del Proyecto de Vicerrectoría de Investigación de la Universidad de Costa Rica \#735B4028.

2 Universidad de Costa Rica, Escuela de Tecnología de Alimentos. San José, Costa Rica. marieguiers@gmail.com (https://orcid.org/00000002-6949-9693); gabriela.davidovich@ucr.ac.cr (autor para la correspondencia; https://orcid.org/0000-0001-6221-4141); eric.wong@ucr. ac.cr (https://orcid.org/0000-0001-9054-130X); elba.cubero@ucr.ac.cr (https://orcid.org/0000-0002-4769-8536).

\section{Resumen}

Introducción. El huevo de gallina (Gallus gallus domesticus) es un alimento nutritivo de la dieta de millones de personas. Su producción mundial se obtiene con el empleo de jaulas convencionales, aunque existen sistemas alternativos como el pastoreo. Objetivo. Comparar la calidad microbiológica, fisicoquímica y el sabor de huevos provenientes de granjas con sistemas productivos convencionales o de pastoreo en Costa Rica. Materiales y métodos. El trabajo se realizó en Alajuela, Costa Rica de octubre del 2016 a mayo del 2017. Se muestrearon superficies y huevos de gallinas Isa Brown en granjas con sistemas productivos convencionales o de pastoreo y se determinó el recuento de coliformes totales, Escherichia coli, mohos y levaduras y ausencia/presencia de Salmonella sp. Además, se analizaron parámetros fisicoquímicos (unidades Haugh, índice de yema, pH, color de yema y dureza de cáscara) y se realizó una prueba sensorial de discriminación con consumidores. Resultados. Los sistemas de producción de pastoreo tienen superficies en contacto con los huevos con recuentos de coliformes totales y E. coli significativamente mayores que las superficies del sistema convencional. Los huevos de pastoreo presentaron recuentos de coliformes totales $\left(3,2 \log _{10} \mathrm{UFC} \mathrm{huevo}^{-1}\right)$ significativamente mayores que los huevos convencionales $\left(2,3 \log _{10} \mathrm{UFC}_{\text {huevo }}{ }^{-1}\right)$. Salmonella sp. estuvo presente en $8 \%$ de las muestras de pastoreo (incidencia no diferente de 0 ) y ausente en las convencionales. Se encontró diferencia en el color de la yema (más oscura en los huevos convencionales). Además, hubo diferencia significativa en el sabor de ambos tipos de huevo. Conclusión. En general, existió diferencia en la calidad microbiológica, fisicoquímica y el sabor de huevos producidos en granja de tipo convencional o pastoreo.

Palabras claves: Escherichia coli, Salmonella sp., Isa Brown, unidades Haugh, prueba tétrada.

\begin{abstract}
Introduction. The hen egg (Gallus gallus domesticus) is a nutritious food in the diet of millions of people. Its worldwide production is obtained from conventional caging systems although alternative grazing systems are also used. Objective. To compare the microbiological and physicochemical quality and flavor of eggs produced under
\end{abstract}


conventional or alternative grazing systems in Costa Rica. Materials and methods. This research was performed in Alajuela, Costa Rica, from October 2016 to May 2017. Environmental surfaces and eggs of Isa Brown hens were sampled on farms from both systems and total coliform, Escherichia coli, yeast and molds counts and absence/presence of Salmonella sp. were determined. Also, physicochemical parameters (Haugh units, yolk index, $\mathrm{pH}$, yolk color, and shell hardness) were measured and a discrimination sensory test with consumers was performed. Results. The grazing production system had surfaces in contact with the eggs with total coliform and E. coli counts significantly higher than those determined for the surfaces of the conventional system. Eggs from grazing hens had total coliform counts $\left(3,2 \log _{10} \mathrm{UFC} \mathrm{egg}^{-1}\right)$ significantly higher than conventional eggs $\left(2,3 \log _{10} \mathrm{UFC} \mathrm{egg}^{-1}\right)$. Salmonella sp. was present in $8 \%$ of the grazing eggs samples (incidence not significantly different from 0 ) and absent in the conventional eggs. A difference was found for the color of the yolk, (darker in conventional eggs). Also, there was a significant difference in the taste of both types of eggs. Conclusion. In general, microbiological and physicochemical quality and flavor of eggs produced under grazing system was different from the ones produced conventionally.

Keywords: Escherichia coli, Salmonella sp., Isa Brown, Haugh units, tetrad method.

\section{Introducción}

El huevo (Gallus gallus domesticus) se encuentra entre los alimentos más nutritivos del mundo y es parte de la dieta saludable de millones de personas (Hui, 2014). La producción mundial de huevos ha aumentado en las últimas décadas, a un ritmo de $4 \%$ anual y tiene un valor de más de 1000000 millones de dólares (Giacomozzi, 2014). En Costa Rica, el huevo es un alimento fundamental en la dieta diaria y su demanda también ha crecido en los últimos años. El consumo de huevo por persona en el país pasó de 190 unidades por año en el 2012 a un promedio de 201 unidades por año en el 2014 (Central America Data, 2014). Del año 2019 al año 2020, Costa Rica aumentó en un $3,16 \%$ la cantidad de gallinas ponedoras y el consumo de huevo en el país fue superior al promedio del resto de Latinoamérica. Se estimó que cada persona consumió 220 huevos en promedio (Ruiz, 2020).

La producción mundial de huevos se obtiene de gallinas ponedoras en jaulas convencionales, debido a los bajos costos de producción; sin embargo, este sistema ha sido criticado por organizaciones que velan por el bienestar animal, así como por los consumidores que cada vez se preocupan más por el origen de sus alimentos (Hannah et al., 2011). Tanto el aumento en la preocupación por el bienestar animal, como la prohibición de la Unión Europea del uso de este tipo de jaulas, han influenciado a los productores de huevo alrededor del mundo a migrar a sistemas alternativos tales como jaulas modificadas y el pastoreo (sin confinamiento en jaulas) (Parisi et al., 2015).

La diferencia entre un sistema de producción convencional y uno alternativo, como el de pastoreo, radica en que en el primero las gallinas se mantienen en jaulas y su alimentación es estándar, mientras tanto, las gallinas en pastoreo son libres de moverse y alimentarse durante todo el día (Sharaf-Eddin et al., 2019; Sulimova et al., 2020).

El huevo es uno de los pocos alimentos que al pasar de la granja al consumidor recibe un tratamiento mínimo, es un alimento perecedero y puede contener una gran variedad de microorganismos patógenos como Salmonella enteritidis y Escherichia coli O157:H7. Estas bacterias patógenas entéricas han sido las causantes de muchos brotes de gastroenteritis alrededor del mundo, debido al consumo de huevos crudos o mal cocinados y han sido aisladas de las cáscaras de huevo, el cual sale de la gallina por el mismo lugar por donde excreta sus heces (Center for Disease Control and Prevention, 2011; Hui, 2014).También, la microbiología del ambiente donde se encuentran los huevos influye en la calidad microbiológica del huevo y, por ende, en la inocuidad del alimento. Es por ello por lo que es de suma importancia conocer la calidad microbiológica del ambiente en ambos tipos de producción de huevos (Jones \& Anderson, 2013). 
Con respecto a la microbiología del huevo, se puede evaluar su calidad e inocuidad con un recuento de mesófilos aerobios, recuento de mohos y levaduras, análisis de coliformes totales, recuento de Staphylococcus aureus y de E. coli y análisis de presencia/ausencia de Salmonella sp. Reglamentos nacionales e internacionales destacan los límites de estos parámetros para huevos aptos para el consumo humano (Poder Ejecutivo, 2006).

Los mohos y levaduras, en general, se utilizan como índice de calidad. Los mohos son capaces de afectar las propiedades sensoriales y reducir el valor nutricional del alimento. Además de esto, sintetizan micotoxinas que no solo pueden tener un impacto negativo en la salud del animal, sino que pueden llegar a contaminar el huevo y afectar a los consumidores (Greco et al., 2014).

El grupo de los coliformes totales se considera un indicador de higiene y sanidad general de los alimentos. Estos son bacilos Gram negativos que pertenecen a la familia de las enterobacterias. Es así como su presencia en alimentos y superficies relacionadas con estos, correlaciona con prácticas de higiene y limpieza (Food and Drug Administration, 2018).

El Reglamento Técnico Centroamericano 67.04.50:17 de "Criterios Microbiológicos para la Inocuidad de Alimentos" establece que debe haber menos de $3 \mathrm{NMP} \mathrm{g}^{-1}$ de E. coli en el interior del huevo. No es aceptable encontrar este microorganismo, aunque sea en el exterior del huevo, debido a que es indicador de contaminación fecal. Esto representa un riesgo de que haya otros patógenos de origen fecal que puedan penetrar al interior del huevo o que ocurra una contaminación cruzada del exterior al interior del huevo a la hora de prepararlo (Food Standards Australia New Zeland, 2016; Poder Ejecutivo, 2018).

A pesar del aumento en la oferta de huevos obtenidos a partir de sistemas alternativos, como el pastoreo, aún falta investigación e información para asegurar metodologías de procesamiento con las que se obtengan huevos que sean inocuos para el consumidor (Jones et al., 2012). Asimismo, hace falta investigación en cuanto a la influencia de los sistemas de producción de huevos en su calidad nutricional, fisicoquímica, microbiológica y su sabor.

Estudios efectuados en Europa han determinado que los huevos producidos en sistemas convencionales presentan mejores características de calidad microbiológica, fisicoquímica y de sabor al compararlos con los huevos obtenidos en sistemas alternativos. Sin embargo, estas características de calidad pueden variar de un país al otro, debido al clima, la raza y la edad de la gallina, entre otros (Englmaierová et al., 2014). Otros estudios han determinado que los huevos provenientes de sistemas alternativos presentan mayor contaminación por bacterias aerobias que los obtenidos por sistemas convencionales; estos afirman que, en los casos en que la gallina tenía mayor contacto con los huevos después de ponerlos, la contaminación microbiológica en la cáscara del huevo era aún mayor (De Reu et al., 2008; Parisi et al., 2015).

Con el aumento en la demanda de este tipo de huevos, resulta importante efectuar estudios como este para conocer las diferencias en su microbiología y garantizar que no representen un peligro para el consumidor. Al entender las diferencias en su microbiología se pueden llegar a desarrollar mejores y más efectivos métodos de manipulación y comercialización con el fin de mejorar su inocuidad (Jones et al., 2011). Se han realizado muy pocos estudios para comparar las diferencias en su calidad fisicoquímica y en el sabor, por lo que la información al respecto es limitada. A pesar de esto, los consumidores aseguran que pueden detectar diferencias en el sabor, olor y color de la yema entre estos tipos de huevo (Bray \& Ankeny, 2017; Jaramillo et al., 2018; Kenji, 2010).

La calidad fisicoquímica del huevo puede ser evaluada por medio de la determinación de distintos parámetros tales como el peso, porcentaje de yema, porcentaje de clara, unidades Haugh (factor relacionado con la viscosidad de la clara del huevo), grosor de cáscara, dureza de cáscara, pH, entre otros (Englmaierová et al., 2014; Yenice et al., 2016).

Las Unidades Haugh son uno de los criterios de calidad fisicoquímica más importantes de la industria avícola. Mide la calidad interna del huevo, con base en la calidad proteínica del mismo y su frescura, al correlacionar la altura de la clara con el peso del huevo. Los menores valores obtenidos están relacionados con un huevo de menor calidad y frescura (Eke et al., 2013). El índice de yema es también un índice de frescura del huevo. Un mayor valor 
evidencia una mayor calidad interna del huevo y una mayor frescura. Este es una relación entre la altura y diámetro de la yema y su valor disminuye conforme se deteriora el huevo (Eke et al., 2013).

El pH de la clara de un huevo recién puesto es de entre 7,6 y 8,5; sin embargo, este aumenta durante su almacenamiento debido a la pérdida de $\mathrm{CO}_{2}$ a través de los poros de la cáscara y puede llegar hasta 9,7. $\mathrm{El} \mathrm{pH} \mathrm{de}$ la yema de un huevo fresco se encuentra cercano a 6,0 y durante el almacenamiento puede alcanzar un valor de 6,4 a 6,9 . El pH de la clara y de la yema aumentan conforme disminuye la frescura y la calidad del huevo (Coutts $\&$ Wilson, 2007).

La calidad de la cáscara es uno de los principales factores que afectan la industria del huevo, ya que esta debe ser lo suficientemente fuerte para evitar que se rompa durante el empaque y transporte y evitar así pérdidas económicas. Una de las formas de medir esta calidad es por medio de la prueba de dureza de cáscara (Ketta \& Tůmová, 2016).

En algunos países se han realizado estudios en los que se compara la calidad fisicoquímica y microbiológica de los huevos producidos bajo estos dos sistemas productivos, sin embargo, estos resultados no se pueden extrapolar a los huevos producidos en Costa Rica, debido a que estos factores pueden ser afectados por las condiciones de producción locales. La información generada en esta investigación permite conocer si existen diferencias entre los dos tipos de huevo producidos en Costa Rica en cuanto a su microbiología, características fisicoquímicas y de sabor. Al entender las diferencias en su microbiología, se puede mejorar su manipulación y comercialización, para así brindar información valiosa a los productores y consumidores de estos huevos. Con base en lo antes descrito, en esta investigación se planteó el objetivo de comparar la calidad microbiológica, fisicoquímica y el sabor de huevos provenientes de granjas con sistemas productivos convencionales o pastoreo en Costa Rica.

\section{Materiales y métodos}

\section{Análisis de superficies de las granjas}

\section{Muestras}

Se tomaron muestras de las superficies de dos granjas de producción de huevo de gallina (Gallus gallus domesticus), una granja de tipo convencional y una de pastoreo, ambas ubicadas en Alajuela, Costa Rica. Como muestra de superficie se entiende aquella que representa un área superficial en el ambiente en el que crece y vive la gallina; estas superficies son las que pueden entrar en contacto directo con la gallina y con el huevo. Se tomaron muestras de cinco superficies distintas en cada ubicación. En la granja de tipo convencional, se muestreó el inicio y el final de la banda recolectora de huevos, el comedero, la segunda banda transportadora de huevos y la reja del suelo de la jaula. En la granja de pastoreo se muestreó el comedero, la canasta utilizada para la recolección de los huevos, la paja de los nidos, el suelo de los nidos y la reja a la entrada de los nidos. Cada sitio, unidad experimental en este caso, se muestreó por duplicado en cada visita y se seleccionó de forma aleatoria.

Las granjas donde se realizaron los muestreos pertenecen a una de las principales empresas productoras de huevo del país y ambas tienen gallinas Isa Brown. La recolección y análisis de estas muestras se realizó en octubre y noviembre del año 2016 (estación lluviosa), se visitó cada granja una vez por semana durante tres semanas consecutivas. Se realizaron tres repeticiones independientes del experimento. Los puntos de muestreo fueron diferentes para cada tipo de producción, debido a las diferencias en su tecnología y operaciones. Para poder comparar la microbiología de ambos tipos de producción, se agruparon los datos obtenidos para cada tipo de producción en tres operaciones: alimentación, transporte y postura.

El procedimiento de toma de la muestra se basó en Jones et al. (2011), por medio del cual se recolectaron las muestras con una gasa estéril de 10 x $10 \mathrm{~cm}$ humedecida con $20 \mathrm{~mL}$ de buffer fosfato salino (PBS) estéril de un área 
de $5 \mathrm{~cm}$ x $10 \mathrm{~cm}$. Después de pasar la gasa por cada punto de muestreo, se colocó en una bolsa de muestra estéril y se agregaron $30 \mathrm{~mL}$ de PBS a cada muestra para homogenizarla por un minuto. Las muestras se mantuvieron en refrigeración y al día siguiente de su recolección se analizaron.

\section{Análisis}

Se analizaron las muestras para determinar las siguientes variables respuesta: recuento de coliformes totales y E. coli (Association of Official Agricultural Chemists, 2005b), y recuento de mohos y levaduras (Pouch \& Ito, 2001) sin modificaciones.

\section{Análisis de huevos comerciales}

\section{Muestras}

Para los análisis de recuentos microbiológicos y de análisis fisicoquímicos, se tomaron muestras de huevos de producción convencional y de pastoreo en diferentes supermercados del área metropolitana. Se eligieron las tres empresas de mayor producción de huevo en el país, que tuvieran los dos tipos de producción (convencional y de pastoreo) y su producto en estos supermercados. Se realizaron tres muestreos en los que se recolectó un paquete de huevos de cada una de las empresas, tanto de huevo convencional como de pastoreo, todos con la misma fecha de empaque. Los muestreos consistieron en las tres repeticiones independientes del experimento. La selección de los huevos del paquete se hizo de manera aleatoria. Las muestras se mantuvieron a temperatura ambiente y no se sometieron a ningún tipo de limpieza, lavado o desinfección. Para el análisis de Salmonella sp. se realizó un procedimiento similar a lo antes descrito, con la modificación que se incluyó una cuarta empresa de huevo convencional y de pastoreo. En este caso cada una de las empresas se consideró una repetición independiente del experimento. Por último, para la prueba de sabor, se usaron huevos de una sola empresa con producción convencional y de pastoreo.

La recolección y análisis de estas muestras se realizó durante el período de diciembre 2016 a mayo 2017.

\section{Análisis}

Para la preparación de las muestras se siguió el procedimiento desarrollado por Moats (1980) y Musgrove et al. (2009), por medio del cual se colocó el huevo con la cámara de aire hacia arriba, se quebró cada huevo con una pinza estéril y se descartó su contenido, se limpió el interior del huevo con una gasa estéril y humedecida con una solución tamponada de fosfatos (PBS por sus siglas en inglés) con el fin de eliminar la clara adherida, se colocó las cáscaras de los tres huevos (unidad experimental) en una bolsa estéril y se quebraron. Por último, se agregó 60 $\mathrm{mL}$ de PBS estéril y se masajeó la muestra en el homogenizador por dos minutos. La selección de las muestras de huevos según el análisis a realizar se asignó de manera aleatoria.

Para comparar las características fisicoquímicas de los huevos de los dos tipos de producción se utilizaron los análisis que se describen a continuación. En todos los casos, las mediciones se realizaron por triplicado, con excepción del análisis de dureza de la cáscara, para el cual se hicieron cinco réplicas de la medición. El cálculo de las unidades Haugh se realizó por medio de la siguiente fórmula (ecuación 1):

$$
\text { Unidades Haugh }=100 \cdot \log \left(H+7,57-1,7 W^{0,37}\right)
$$

Donde $\mathrm{W}=$ peso del huevo (g) y H= altura del espesor de la clara de huevo (mm).

El huevo se quebró sobre una lámina de vidrio que se colocó sobre una superficie blanca y se determinó el peso 
en balanza analítica, mientras que la altura de la clara se determinó con un vernier en tres puntos distintos de esta (Gjorgovska et al., 2011). Para medir el pH se separaron la clara y la yema, y se utilizó un pHímetro con electrodo de vidrio calibrado (Association of Official Agricultural Chemists, 2005a). La dureza de la cáscara del huevo o resistencia a la ruptura se evaluó con el texturómetro (Ta.XTPlus-Texture Analyzer de Stable Micro Systems Ltd.), por medio de la compresión uniaxial de la región central de cada huevo. Cada huevo se colocó en una plataforma y se perforó con un cilindro de $3 \mathrm{~mm}$ a una velocidad constante de prueba y pre-prueba de $5 \mathrm{~mm} \mathrm{~s}^{-1}$ y una velocidad post prueba de $40 \mathrm{~mm} \mathrm{~s}^{-1}$. La celda utilizada tenía una carga de $50 \mathrm{~kg}$ en modo de compresión y una distancia de $3 \mathrm{~mm}$. La fuerza que se requirió para romper la cáscara se registró como la resistencia de la cáscara a la ruptura (N) (Caner \& Yuceer, 2015). El color de la yema se determinó por medio de la escala de color de yema DSM de la compañía Roche. Los huevos se quebraron sobre una lámina de vidrio que se colocó sobre una superficie blanca y con fuente de luz adecuada con el fin de no alterar la medición de color, luego se procedió a comparar el color de la yema de cada muestra con la escala de color (Gjorgovska et al., 2011). En la medición del índice de yema, el huevo se quebró sobre una lámina de vidrio que se colocó sobre una superficie blanca. Se utilizó un vernier para determinar la altura y el diámetro de la yema cruda (Gjorgovska et al., 2011). El cálculo se realizó por medio de la siguiente fórmula (ecuación 2):

$$
\text { Índice de yema }=\frac{\text { Altura de yema }(\mathrm{mm})}{\text { Diámetro de yema }(\mathrm{mm})} \times 100
$$

Para comparar la presencia de Salmonella en ambos tipos de producción, la unidad experimental consistió en $25 \mathrm{~g}$ de cáscaras de huevo. El análisis se realizó por el método de cultivo con la confirmación bioquímica en API 20E (Food and Drug Administration, 2018).

Para determinar posibles diferencias en el sabor de los huevos de cada tipo de producción se aplicó una prueba tétrada a 96 personas (Ennis \& Jesionka, 2011). Se presentaron a los panelistas dos muestras de cada tipo de huevo que consistieron en trozos de $2 \mathrm{~cm}$ x $2 \mathrm{~cm}$ de tortas de huevo preparadas con $100 \mathrm{~g}$ de huevo y $1 \%$ de sal, en un ambiente de luz roja para valorar su sabor.

\section{Análisis estadísticos}

Con los resultados de las muestras de superficies de las granjas se hicieron dos análisis estadísticos con los mismos datos. Para cada tipo de producción y variable respuesta se realizó un análisis de varianza (ANDEVA), seguido de la prueba de Tukey, con base en un diseño irrestricto aleatorio de cinco tratamientos con el fin de determinar diferencias debidas a los sitios de muestreo. Este análisis y todos los de esta investigación se trabajaron con el paquete estadístico JMP 5.0.1.2. (SAS Institute, NC, Estados Unidos) a un nivel de significancia del $5 \%$.

Con el fin de comparar las variables respuesta para ambos tipos de producción cuyos sitios muestreados no fueron los mismos, se realizó una agrupación de los sitios en tres operaciones generales: alimentación, transporte y postura, con un diseño irrestricto aleatorio con arreglo factorial 2 X 3 (dos niveles de tipo de producción y los tres niveles del sitio muestreado), el cual se analizó mediante un ANDEVA y posterior prueba de Tukey.

Para comparar los recuentos de coliformes totales, E. coli y mohos y levaduras, así como los parámetros fisicoquímicos, se utilizó un ANDEVA para un diseño de bloques al azar con dos tratamientos (convencional y pastoreo), donde el bloque correspondió a cada uno de los tres lotes de huevos analizados y las repeticiones a cada una de las tres empresas, por tipo de producción, seleccionadas. Los datos de ausencia o presencia de Salmonella se analizaron mediante regresión logística para probar si la proporción de muestras positivas era diferente en cada tipo de producción. 
El análisis de la prueba tétrada para sabor se basó en una potencia de prueba de 0,9 , un delta de 1,10 y un alfa de 0,01 (Ennis \& Jesionka, 2011). Se realizó el cálculo de la probabilidad exacta para una distribución binomial con el paquete XLSTAT (Addinsoft, París, Francia).

\section{Resultados}

\section{Caracterización y comparación de parámetros microbiológicos en muestras de superficies de sistemas de producción convencional y pastoreo}

En el sistema de producción convencional no se encontró diferencia significativa $(\mathrm{p}>0,05)$ en los recuentos de coliformes totales y E. coli para los distintos sitios de muestreo (Cuadro 1). Los mohos y levaduras se encontraron en el orden de 2 a $6 \log$ UFC $\mathrm{cm}^{-2}$, donde el inicio, el fin de banda y el comedero presentaron los recuentos más altos $(\mathrm{p}<0,05)$, seguido por la segunda banda y la reja del piso de la jaula.

Cuadro 1. Recuentos de coliformes totales, E. coli y mohos y levaduras en muestras de superficies según sitio de muestreo para el sistema de producción convencional de huevo de gallina (Gallus gallus domesticus). Escuela de Tecnología de Alimentos de la Universidad de Costa Rica. Alajuela, Costa Rica, 2016.

Table 1. Counts of total coliforms, E. coli and yeasts and molds in surface samples according to sampling site for the conventional production system of hen egg (Gallus gallus domesticus). School of Food Technology, Universidad de Costa Rica. Alajuela, Costa Rica, 2016.

\begin{tabular}{lccc}
\hline Sitio de muestreo & $\begin{array}{c}\text { Recuento de coliformes } \\
\text { totales }\left(\log \mathbf{U F C ~} \mathbf{~ c m}^{-2}\right)\end{array}$ & $\begin{array}{c}\text { Recuento de Escherichia coli } \\
\left(\log \mathbf{U F C} \mathbf{~ c m}^{-2}\right)\end{array}$ & $\begin{array}{c}\text { Recuento de mohos y } \\
\text { levaduras }\left(\mathbf{l o g} \mathbf{U F C} \mathbf{~ c m}^{-2}\right)\end{array}$ \\
\hline Inicio banda & $1,8 \pm 0,3$ & $1,3 \pm 0,3$ & $5,4 \pm 0,2^{\mathrm{a}}$ \\
Fin banda & $1,6 \pm 0,5$ & $1,0 \pm 0,2$ & $5,2 \pm 0,5^{\mathrm{a}}$ \\
Segunda banda & $1,8 \pm 0,4$ & $0,95 \pm 0,00$ & $3,4 \pm 0,5^{\mathrm{b}}$ \\
Reja piso jaula & $1,4 \pm 0,3$ & $1,04 \pm 0,07$ & $1,9 \pm 0,1^{\mathrm{c}}$ \\
Comedero & $1,3 \pm 0,7$ & $0,97 \pm 0,02$ & $6 \pm 1^{\mathrm{a}}$ \\
\hline
\end{tabular}

Recuentos con letras diferentes en cada columna presentan diferencias significativas entre sí $(\mathrm{p}<0,05)$. Valores sin letras asociadas en cada columna no presentan diferencias significativas entre sí $(\mathrm{p}>0,05)$. / Counts with different letters in each column show significant differences between them $(\mathrm{p}<0.05)$. Values with no uppercase letter associated in each column are not significantly different between them $(\mathrm{p}>0.05)$.

Se muestran los promedios $(n=3)$ con el respectivo intervalo de confianza $(\alpha=0,05)$. / Values shown are means $(n=3)$ with corresponding confidence interval $(\alpha=0.05)$.

En el sistema de producción de pastoreo se encontró diferencia significativa $(\mathrm{p}<0,05)$ en los recuentos de coliformes totales y E. coli, que variaron entre 1 a 6 y 1 a $5 \log \mathrm{UFC} \mathrm{cm}^{-2}$, respectivamente (Cuadro 2). En ambos casos, la paja del nido fue la muestra con el recuento más alto y diferente a los otros sitios. Los recuentos de mohos y levaduras también presentaron diferencias significativas $(\mathrm{p}<0,05)$ con valores entre 3 a $6 \log \mathrm{UFC} \mathrm{cm}^{-2}$. La paja del nido presentó la mayor cantidad de este indicador de calidad, seguido por la canasta de transporte y la reja a la entrada del nido. 
Cuadro 2. Recuentos de coliformes totales, E. coli y mohos y levaduras en muestras de superficies según sitio de muestreo para el sistema de producción por pastoreo de huevo de gallina (Gallus gallus domesticus). Escuela de Tecnología de Alimentos de la Universidad de Costa Rica. Alajuela, Costa Rica, 2016.

Table 2. Counts of total coliform, E. coli and yeasts and molds in surface samples according to sampling site for the grazing production system of hen egg (Gallus gallus domesticus). School of Food Technology, Universidad de Costa Rica. Alajuela, Costa Rica, 2016.

\begin{tabular}{lccc}
\hline Sitio de muestreo & $\begin{array}{c}\text { Recuento de coliformes totales } \\
\left(\log \mathbf{U F C} \mathbf{~ m}^{-2}\right)\end{array}$ & $\begin{array}{c}\text { Recuento de Escherichia coli } \\
\left(\log \mathbf{U} \mathbf{C} \mathbf{~ m}^{-2}\right)\end{array}$ & $\begin{array}{c}\text { Recuento de mohos y levaduras } \\
\left(\log \mathbf{U F C} \mathbf{~ c m}^{-2}\right)\end{array}$ \\
\hline Canasta transporte & $3,1 \pm 0,6^{\mathrm{a}}$ & $1,49 \pm 0,08^{\mathrm{a}}$ & $4,6 \pm 0,4^{\mathrm{ab}}$ \\
Nido & $2,9 \pm 0,9^{\mathrm{a}}$ & $2,0 \pm 0,9^{\mathrm{a}}$ & $4,1 \pm 0,4^{\mathrm{bc}}$ \\
Paja del nido & $5,7 \pm 0,1^{\mathrm{b}}$ & $5,0 \pm 0,2^{\mathrm{b}}$ & $5,7 \pm 0,2^{\mathrm{a}}$ \\
Reja & $2 \pm 1^{\mathrm{a}}$ & $1,7 \pm 0,6^{\mathrm{a}}$ & $3,2 \pm 0,1^{\mathrm{c}}$ \\
Comedero & $1,5 \pm 0,3^{\mathrm{a}}$ & $0,95 \pm 0,00^{\mathrm{a}}$ & $3,9 \pm 0,8^{\mathrm{bc}}$ \\
\hline
\end{tabular}

Recuentos con letras diferentes en cada columna presentan diferencias significativas entre sí $(\mathrm{p}<0,05)$. Se muestran los promedios ( $\mathrm{n}=3$ ) con el respectivo intervalo de confianza $(\alpha=0,05)$. / Counts with different letters in each column show significant differences between them $(\mathrm{p}<0.05)$. Values shown are means $(n=3)$ with corresponding confidence interval $(\alpha=0.05)$.

Al comparar los dos tipos de producción se agruparon los sitios en operaciones similares (Cuadro 3). Se determinó que el efecto de los sitios sobre los recuentos de coliformes totales, E. coli y mohos y levaduras dependieron del tipo de producción $(\mathrm{p}<0,0001)$. En general, los recuentos de los sitios relacionados con el transporte, la postura y la alimentación fueron más bajos (de 1 a $1,5 \log \mathrm{UFC} \mathrm{cm}^{-2}$ ) en el caso del sistema de producción convencional en comparación con el sistema de producción de pastoreo. En el sistema convencional no se encontraron diferencias en los tres índices microbiológicos según el tipo de operación, mientras que en pastoreo sí $(\mathrm{p}<0,05)$. Los recuentos más altos obtenidos fueron para la operación de transporte y postura.

Cuadro 3. Recuentos de coliformes totales, E. coli y mohos y levaduras en muestras de superficies según tipo de producción de huevo de gallina (Gallus gallus domesticus) y operación del proceso. Escuela de Tecnología de Alimentos de la Universidad de Costa Rica. Alajuela, Costa Rica, 2016.

Table 3. Counts of total coliform, E. coli and yeasts and molds in surface samples according to hen egg (Gallus gallus domesticus) type of production system and process operation. School of Food Technology, Universidad de Costa Rica. Alajuela, Costa Rica, 2016.

\begin{tabular}{lcccc}
\hline Tipo producción & Operación & $\begin{array}{c}\text { Recuento de coliformes } \\
\text { totales }\left(\log \text { UFC } \mathbf{~ c m}^{-2}\right)\end{array}$ & $\begin{array}{c}\text { Recuento de Escherichia } \\
\text { coli }\left(\mathbf{l o g} \text { UFC } \mathbf{~ c m}^{-2}\right)\end{array}$ & $\begin{array}{c}\text { Recuento de mohos y } \\
\text { levaduras }\left(\log \text { UFC } \mathbf{c m}^{-2}\right)\end{array}$ \\
\hline Convencional & Transporte & $1,7 \pm 0,3^{\mathrm{b}}$ & $1,1 \pm 0,1^{\mathrm{b}}$ & $4,7 \pm 0,3^{\mathrm{ab}}$ \\
& Postura & $1,4 \pm 0,3^{\mathrm{b}}$ & $1,04 \pm 0,07^{\mathrm{b}}$ & $1,9 \pm 0,1^{\mathrm{c}}$ \\
& Alimentación & $1,3 \pm 0,7^{\mathrm{b}}$ & $0,97 \pm 0,02^{\mathrm{b}}$ & $6 \pm 1^{\mathrm{a}}$ \\
Pastoreo & Transporte & $3,1 \pm 0,6^{\mathrm{a}}$ & $1,49 \pm 0,08^{\mathrm{b}}$ & $4,6 \pm 0,4 \mathrm{a}^{\mathrm{b}}$ \\
& Postura & $3,7 \pm 0,5^{\mathrm{a}}$ & $2,9 \pm 0,5^{\mathrm{a}}$ & $4,3 \pm 0,2^{\mathrm{b}}$ \\
& Alimentación & $1,5 \pm 0,3^{\mathrm{b}}$ & $0,95 \pm 0,00^{\mathrm{b}}$ & $3,9 \pm 0,8^{\mathrm{b}}$ \\
\hline
\end{tabular}

Recuentos con letras diferentes en cada columna presentan diferencias significativas entre sí $(\mathrm{p}<0,05)$. / Counts with different letters in each column show significant differences between them $(\mathrm{p}<0.05)$.

Se muestran los promedios $(n=3)$ con el respectivo intervalo de confianza $(\alpha=0,05)$. / Values shown are means $(n=3)$ with corresponding confidence interval $(\alpha=0.05)$. 


\section{Comparación de parámetros microbiológicos en muestras de huevo de sistemas de producción convencional y pastoreo}

En cuanto a las muestras de huevo (Cuadro 4), se determinó que los huevos provenientes de granjas con sistema de pastoreo presentaron recuentos significativamente mayores $(\mathrm{p}<0,05)$ de coliformes totales que aquellos provenientes de sistemas convencionales. No se obtuvieron diferencias significativas en los recuentos de E. coli y mohos y levaduras para ambos tipos de huevos.

Cuadro 4. Recuentos de coliformes totales, E. coli y mohos y levaduras en la cáscara de huevo de gallina (Gallus gallus domesticus) según tipo de producción. Escuela de Tecnología de Alimentos de la Universidad de Costa Rica. Alajuela, Costa Rica, 2016.

Table 4. Counts of total coliform, E. coli and yeasts and molds count in the shell of hen egg (Gallus gallus domesticus) according to type of production. School of Food Technology, Universidad de Costa Rica. Alajuela, Costa Rica, 2016.

\begin{tabular}{|c|c|c|c|}
\hline Tipo de producción & $\begin{array}{l}\text { Recuento de coliformes } \\
\text { totales }(\log \text { UFC huevo-1) }\end{array}$ & $\begin{array}{l}\text { Recuento de Escherichia coli } \\
\left(\log \mathrm{UFC}^{\text {huevo-1 }}\right)\end{array}$ & $\begin{array}{c}\text { Recuento de mohos y } \\
\text { levaduras (log UFC huevo-1) }\end{array}$ \\
\hline Convencional & $2,25 \pm 0,00^{\mathrm{a}}$ & $2,25 \pm 0,00$ & $4,2 \pm 0,7$ \\
\hline Pastoreo & $3,2 \pm 0,7^{b}$ & $2,7 \pm 0,6$ & $4,0 \pm 0,3$ \\
\hline
\end{tabular}

Recuentos con letras diferentes en cada columna presentan diferencias significativas entre sí $(\mathrm{p}<0,05)$. / Counts with different letters in each column show significant differences between them $(\mathrm{p}<0.05)$.

Valores sin letras asociadas en cada columna no presentan diferencias significativas entre sí ( $\mathrm{p}>0,05)$. / Values with no uppercase letter associated in each column are not significantly different from others $(\mathrm{p}>0.05)$.

Se muestran los promedios $(n=3)$ con el respectivo intervalo de confianza $(\alpha=0,05)$. / Values shown are means $(n=3)$ with corresponding confidence interval $(\alpha=0.05)$.

El $8 \%$ de las muestras de huevo provenientes del sistema de pastoreo fueron positivas para Salmonella sp., mientras que de los huevos del sistema convencional no fue posible aislar este patógeno. No hubo diferencia estadística en la proporción de huevos positivos por Salmonella sp. entre ambos tipos de producción ( $\mathrm{p}=0,9731$ ), es decir, no es estadísticamente diferente de cero.

\section{Comparación de parámetros fisicoquímicos y sabor en muestras de huevo de sistemas de producción convencional y pastoreo}

A las muestras de huevo se les midieron varios parámetros fisicoquímicos con el fin de poder comparar su calidad y relacionarla con las condiciones de producción (Cuadro 5).

El color de yema fue el único parámetro en el que se encontraron diferencias significativas entre los dos tipos de huevos. Cabe destacar que los colores de esta escala van desde un amarillo claro a un anaranjado oscuro. Las yemas de los huevos obtenidos en sistema convencional presentaron un color significativamente más oscuro $(\mathrm{p}<0,05)$ que el de las yemas de los huevos de pastoreo, lo que significa que su coloración fue más anaranjado que amarillo.

En la prueba tétrada para medir la posible diferencia de sabor, de los 96 panelistas, hubo 37 aciertos y se obtuvo una probabilidad exacta de 0,0435 de encontrar la diferencia. Con un 95,7 \% de confianza, existieron diferencias significativas en el sabor de los huevos producidos en ambos sistemas productivos. 
Cuadro 5. Mediciones de parámetros fisicoquímicos de huevos de gallina (Gallus gallus domesticus), según tipo de producción. Escuela de Tecnología de Alimentos de la Universidad de Costa Rica. Alajuela, Costa Rica, 2017.

Table 5. Measures for physicochemical parameters in hen eggs (Gallus gallus domesticus) according to production type. School of Food Technology, Universidad de Costa Rica. Alajuela, Costa Rica, 2017.

\begin{tabular}{lcccccccc}
\hline Tipo de producción & $\begin{array}{c}\text { Unidades } \\
\text { Haugh }\end{array}$ & $\begin{array}{c}\text { Índice de } \\
\text { yema }\end{array}$ & Color yema & pH clara & pH yema & pH & Dureza (N) \\
\hline Convencional & 74 & 38 & 11 & 9,23 & 6,24 & 8,16 & 32558 \\
& \pm 4 & \pm 2 & $\pm 0^{\mathrm{a}}$ & $\pm 0,05$ & $\pm 0,06$ & $\pm 0,07$ & \pm 1663 \\
\hline Pastoreo & 74 & 37 & 10,2 & 9,27 & 6,22 & 8,20 & 34000 \\
& \pm 4 & \pm 1 & $\pm 0,4^{\mathrm{b}}$ & $\pm 0,07$ & 0,05 & $\pm 0,06$ & \pm 2997 \\
\hline
\end{tabular}

Valores con letras diferentes en cada columna presentan diferencias significativas entre sí $(\mathrm{p}<0,05)$. / Values with different letters in each column show significant differences between them $(\mathrm{p}<0.05)$.

Valores sin letras asociadas en cada columna no presentan diferencias significativas entre sí $(\mathrm{p}>0,05)$. / Values with no uppercase letter associated in each column are not significantly different from others $(\mathrm{p}>0.05)$.

Se muestran los promedios $(n=3)$ con el respectivo intervalo de confianza $(\alpha=0,05)$. / Values shown are means $(n=3)$ with corresponding confidence interval $(\alpha=0.05)$.

\section{Discusión}

Se caracterizaron las condiciones higiénicas ambientales de los dos sistemas productivos en estudio, para obtener información de apoyo para la comparación de la calidad microbiológica, fisicoquímica y el sabor de huevos provenientes de granjas con sistemas productivos convencionales o pastoreo en Costa Rica.

En el sistema de producción convencional, los coliformes totales, indicadores de sanidad en general, estuvieron en el orden de 1-2 log UFC $\mathrm{cm}^{-2}$, lo que indica un ambiente limpio. Los recuentos de E. coli, indicador de contaminación fecal, también se consideraron bajos (en el orden de $1 \log \mathrm{UFC} \mathrm{cm}^{-2}$ ) y normales tratándose de ambientes en contacto con el animal y sus heces.

En el sistema de producción de pastoreo, a diferencia del sistema convencional, los niveles de coliformes totales y E. coli sugirieron condiciones de sanidad más comprometidas, mientras que los niveles de mohos y levaduras fueron similares. Al comparar los dos tipos de producción se agruparon los sitios en operaciones similares, se determinó que el efecto de los sitios sobre los recuentos de coliformes totales, E. coli y mohos y levaduras, dependieron del tipo de producción. Es así como hubo recuentos mayores en la producción de pastoreo que en la convencional.

Los resultados coinciden con lo reportado por De Reu et al. (2008), quienes indicaron que las galeras con suelo de paja o granza tienen hasta nueve veces más bacterias que las galeras con jaulas y piso de alambre, dada la acumulación de polvo, insectos, suciedad en general y heces de las gallinas en las primeras. Otro estudio similar llegó a la conclusión de que la separación del animal de sus heces en el sistema convencional de jaulas, reduce el riesgo de contaminación fecal en los ambientes (Fischer, 2010). También, en otro estudio se analizó el ambiente de ambos tipos de sistemas y obtuvieron mayores recuentos totales de aerobios y de coliformes en los sistemas alternativos (aviario y jaulas enriquecidas) que en los sistemas de jaulas convencionales (Jones et al., 2015). En una investigación de estos mismos autores se demostró que el ambiente de un sistema de pastoreo contiene mayor número de microrganismos y una mayor diversidad de ellos, debido a una mayor exposición al ambiente externo donde hay heces, tierra, roedores, entre otros (Jones et al., 2012). En contraste con lo anterior, se reportó que no hubo diferencias significativas entre los recuentos bacterianos de las bandas de transporte de ambos sistemas de producción ni de las cajas o nidos donde se ponen los huevos (Wilson et al., 2021). 
La operación de alimentación del sistema convencional fue la que presentó el mayor recuento de mohos y levaduras. El alto recuento que se obtuvo de la muestra del comedero del sistema convencional evidenció que hubo un problema de limpieza en los silos de almacenamiento del alimento, canoas de transporte o el comedero. Esto puede contaminar el alimento de la gallina y llegar a afectar su salud y bajar su productividad o incluso contaminar los huevos y enfermar a los consumidores (Greco et al., 2014).

En cuanto a las muestras de huevo analizadas, el recuento de coliformes totales reflejó un riesgo con un potencial mayor de contaminación en el interior del huevo. El Poder Ejecutivo (2006), RTCR 397:2006, señaló como valor máximo de este recuento en el interior del huevo $3 \log \mathrm{UFC}^{-1}$. Se espera que la contaminación y, por ende, el recuento de coliformes totales sea aún mayor en el exterior. Como se puede observar, en el exterior de los huevos analizados se obtuvieron recuentos de 2 y $3 \log$ UFC de coliformes totales por huevo convencional y de pastoreo, respectivamente. Si se compara con los resultados que permite la normativa nacional, se puede decir que los huevos analizados no presentaron recuentos inaceptables de coliformes totales en su cáscara pues los valores no fueron mayores que los límites permitidos para el interior del huevo (Poder Ejecutivo, 2006).

En cuanto a los recuentos de E. coli, se considera insatisfactorio cuando estos son mayores a $2 \log \mathrm{UFC} \mathrm{g}^{-1}$ en alimentos crudos, pues indica que hay un riesgo potencial de contaminación de origen fecal por prácticas de higiene inadecuadas, contaminación cruzada o un procesamiento inadecuado (Food Standards Australia New Zeland, 2016). Los recuentos obtenidos en la cáscara de ambos tipos de huevos se encontraron por encima de este valor, lo que indica contaminación fecal. Esto representa un riesgo de que haya otros patógenos de origen fecal que puedan penetrar al interior del huevo o que ocurra una contaminación cruzada del exterior al interior del huevo a la hora de prepararlo (Food Standards Australia New Zeland, 2016). Es por esto que se deben aumentar los controles en el procesamiento, mejorar las prácticas de higiene, limpieza y desinfección, así como las prácticas de higiene de los manipuladores para evitar que estos recuentos sean insatisfactorios.

No hubo diferencia estadística en la proporción de huevos positivos por Salmonella sp. entre ambos tipos de producción, pero a nivel cualitativo es importante considerar que algunas muestras del sistema de pastoreo contenían el patógeno y pueden representar riesgo de enfermedad para los consumidores (Hui, 2014). En comparación, el estudio de Wilson et al. (2021) encontró un $37 \%$ de presencia de Salmonella enterica en las muesras de superficies tomadas del sistema de producción convencional, mientras que en el sistema de pastoreo todas las muestras presentaron ausencia de este patógeno.

Los resultados de las muestras de huevo también concordaron con diversos estudios que se han realizado en otros países, tales como el de De Reu (2006) quien encontró mayores recuentos de bacterias mesófilas aerobias, mientras que Parisi et al. (2015) obtuvieron mayores recuentos de enterobacterias en huevos provenientes de sistemas alternativos con respecto a los huevos de gallinas en jaulas convencionales. El tipo de sistema influye significativamente en el recuento de bacterias aerobias, Enterococcus y E. coli en la cáscara del huevo (Englmaierová et al., 2014). Los huevos de sistemas convencionales de jaulas obtuvieron los recuentos de E. coli más bajos, mientras que los de sistemas alternativos tuvieron recuentos mayores en hasta 2 log. Los huevos con mayor contaminación fueron aquellos que se pusieron en paja o granza. Resultados similares se encontraron en estudios sobre mohos, donde se analizó la microbiota de mohos en huevos de distintos sistemas de producción. El estudio indicó que los huevos de pastoreo tenían una mayor diversidad de esta microbiota que los del sistema convencional, ya que la posibilidad de controlar las condiciones ambientales es muy baja y las gallinas tienen acceso libre con distintas fuentes de contaminación. En el caso de los huevos de las gallinas en jaula, se encontró una baja diversidad, con ocho especies de mohos, pero solo dos capaces de producir micotoxinas (Tomczyk et al., 2018).

De manera similar a lo obtenido en este trabajo, otros autores en sus investigaciones, encontraron que los huevos provenientes de gallinas enjauladas sin nido y sin ningún material en el suelo presentaron menores recuentos de coliformes totales y E. coli que los que provenían de gallinas en sistemas alternativos con nidos y aserrín en el suelo (Hannah et al., 2011; Singh et al., 2009). Además, en varias investigaciones E. coli es la bacteria 
que ha sido aislada con mayor frecuencia de la cáscara de huevos sin lavar y en mayor concentración en huevos provenientes de sistemas alternativos como el pastoreo (Jones et al., 2012; Musgrove et al., 2009), lo cual apoya el hallazgo en este estudio. Los resultados de estos investigadores y los de De Reu et al. (2009), coinciden con los de la presente investigación, en que no solo el sistema de producción influye en el grado de contaminación de los huevos, sino que también la infraestructura y manejo de la granja juegan un papel importante en la microbiología de estos. Esto lo concluyeron basado en que encontraron diferencias en la contaminación de la cáscara de huevos provenientes de diferentes granjas con el mismo sistema de producción.

En la presente investigación se determinó alta contaminación fecal en los huevos de pastoreo y presencia de Salmonella sp. en estas muestras. Esto puede asociarse con el hecho de que, en la granja visitada, las gallinas ponían los huevos en el suelo algunas veces y estos se recogían igual que los demás. Cabe destacar que uno de los principales problemas en los sistemas alternativos y, en especial el de pastoreo, es que las gallinas ponen los huevos no solo en el nido sino también en el suelo que se encuentra cubierto por un material como paja o granza que acumula heces y otros contaminantes. En concordancia con esto resultados, De Reu (2006) y Protais et al. (2003) encontraron que los huevos que son puestos en el suelo tienen una mayor carga bacteriana (de hasta 7 log) que los que son puestos en los nidos. En ese mismo sentido, Smeltzer et al. (1979) encontraron que los huevos que eran puestos en los suelos sucios de la galera tenían una mayor probabilidad de presentar contaminación en su interior.

Los sistemas convencionales, como el de jaulas tipo batería, tienen la ventaja de que las gallinas están separadas de sus heces de manera muy eficiente. Las jaulas se encuentran colocadas de tal manera que las heces caen a un nivel inferior donde se recolectan. Al no haber nido ni tener ningún material en el suelo de la jaula, no hay acumulación de heces ni de contaminación fecal. Lo anterior se pudo observar en la galera de sistema convencional que se visitó. Además, las jaulas impiden que las gallinas pongan huevos en el suelo (Fischer, 2010; Singh et al., 2009; Svobodová \& Tůmová, 2014). Otro factor que se relaciona con lo anterior y que fue observado en la granja, es el tiempo de permanencia de los huevos en la galera. Se ha encontrado que cuanto mayor tiempo estén los huevos en la galera y en contacto con la gallina, mayor es la oportunidad de que estos se contaminen. Los sistemas convencionales tienen la ventaja que apenas el huevo es puesto, este cae en una banda transportadora. Esta los lleva hacia el área de empaque y, por lo tanto, se reduce el tiempo que la gallina tiene contacto con el huevo y el tiempo que este pasa en la galera (Fronding, 1998).

La contaminación de la cáscara de los huevos del sistema de pastoreo, reflejada en los análisis microbiológicos, se pudo corroborar en las visitas a las granjas, ya que en ocasiones los huevos estaban sucios con heces y los colaboradores debían limpiarlos para removerlas. Esta contaminación no solo se ve afectada por la limpieza de la galera, sino también por la dieta de las gallinas. Las dietas en las que se aumenta la humedad de las heces no solo generan un incremento en la cantidad de huevos contaminados por heces, sino que también aumenta la contaminación microbiana en los huevos con apariencia de limpios y sin excretas en su cáscara (Smith et al., 2000). Las gallinas de ambos tipos de producción consumieron el mismo concentrado en el momento de las visitas, ya que pertenecen a la misma empresa. Sin embargo, la diferencia es que la dieta de las gallinas de pastoreo consta un 20 $\%$ de este concentrado y un $80 \%$ es libre y corresponde a insectos, plantas, granza u otros que puedan encontrar. Esta variabilidad en su alimentación puede aumentar la humedad de las heces de las gallinas de pastoreo y por lo tanto, influir en su contaminación.

Los resultados obtenidos muestran que no se obtuvieron diferencias significativas en las unidades Haugh de los huevos estudiados. Lo anterior puede deberse a que todos los huevos que se muestrearon tenían la misma fecha de empaque, por lo que su frescura era muy similar. Los valores obtenidos cumplieron con lo establecido en el RTCR 397:2006 para "huevos frescos o refrigerados de gallina para el consumo humano", ya que todas las muestras presentaron más de 20 unidades Haugh. Los valores encontrados se consideraron normales y evidenciaron que a los huevos les queda vida útil y tienen adecuada calidad proteínica (Eke et al., 2013; Poder Ejecutivo, 2006). Los resultados obtenidos en estudios publicados por diversos investigadores son contradictorios, ya que mientras unos 
no han encontrado diferencias significativas entre los dos sistemas de producción, otros obtuvieron valores mayores de unidades Haugh en los huevos de gallinas en sistema convencional y otros en los huevos de gallinas de pastoreo (Karcher et al., 2015; Perić et al., 2016; Tůmová \& Ebeid, 2003; Yenice et al., 2016).

Los resultados obtenidos para el índice de yema mostraron que ambos tipos de huevos no presentaron diferencias significativas. Esto significa que la frescura de los huevos de pastoreo no difirió de la de los huevos de gallinas en sistema convencional y, en ambos casos, los valores demostraron que el huevo no se encontraba deteriorado, sino más bien fresco. Este hallazgo concuerda con lo publicado por Jones et al. (2014). De forma contraria, otros estudios reportaron mayores valores de índice de yema en huevos provenientes de sistema de jaulas convencionales y llegaron a la conclusión de que el tipo de producción influye en las características de calidad interna y externa de los huevos (Englmaierová et al., 2014; Yenice et al., 2016).

El resultado del color de yema de los huevos analizados en este estudio, concuerda con lo que reportan diversos autores quienes indicaron que la yema de los huevos de producción convencional es más oscura que la de los huevos de pastoreo, es decir más anaranjada que amarilla (Jaramillo et al., 2018; Mugnai et al., 2009; Perić et al., 2016; Stojanova et al., 2016; Tercic et al., 2012). Lo anterior puede atribuirse a las diferencias en la alimentación de las gallinas en cada uno de los tipos de producción. El concentrado contiene colorantes sintéticos que los productores agregan con el fin de alcanzar el color de yema deseado. El color de la yema es determinado por el contenido y perfil de carotenoides presentes en su alimentación, por lo que esta puede ser modificada con el fin de alcanzar un color objetivo. La proporción de carotenoides en la dieta que son absorbidos y depositados en la yema de huevo determinan su color, el cual va desde un amarillo pálido a un anaranjado oscuro (Bovšková et al., 2014).

Se encontró un estudio que reportó que los huevos de pastoreo analizados presentaron un color de yema más oscuro que los de sistema convencional (Holt et al., 2011). Esto pudo deberse a que las gallinas al basar $80 \%$ de su alimentación en insectos, pasto, granza y plantas, aumentan su ingesta de carotenoides en comparación con las gallinas en sistema convencional. Sin embargo, esto depende de las condiciones de producción, alimentación y manejo que se da en cada país. Es difícil extrapolar a nuestro país los resultados encontrados por investigadores en otros países, debido a la variabilidad en las condiciones de producción, clima, raza, línea genética de gallina, manejo, entre otros.

No se encontraron diferencias significativas entre el $\mathrm{pH}$ total, $\mathrm{pH}$ de la clara y $\mathrm{pH}$ de la yema de ambos tipos de huevos en estudio. Lo anterior pudo deberse a que se utilizaron huevos con la misma fecha de empaque con el fin de garantizar una frescura lo más similar posible (todos con menos de 7 días desde su puesta). Al no haber diferencias en su tiempo de almacenamiento y su frescura, es de esperar que tampoco las haya en su $\mathrm{pH}$. El rango de pH de la clara de los huevos en este estudio fue de 8,73 a 9,34, lo cual indicó que el huevo no era recién puesto, ya que en estos casos el valor de $\mathrm{pH}$ es de entre 7,6 y 8,5. En almacenamiento, el $\mathrm{pH}$ de la clara de un huevo puede llegar hasta 9,7, valor no alcanzado por los huevos analizados. El pH de la yema de los huevos se encontraba en valores entre 6,18 y 6,30 lo cual concuerda con otros estudios que indican que este valor puede alcanzar valores de 6,0 en almacenamiento (Coutts \& Wilson, 2007).

En esta investigación, no se obtuvieron diferencias significativas en los valores de dureza de la cáscara de ambos tipos de huevos. Estudios con estos mismos resultados indicaron que otros factores relacionados con el manejo de las gallinas tienen una mayor influencia en la calidad de la cáscara que el tipo de producción (Clerici et al., 2006; Stojanova et al., 2016). Existen estudios que se diferencian en cuanto a los resultados antes descritos. Se han encontrado cáscaras significativamente más fuertes en los huevos de gallinas de jaulas convencionales (Englmaierová et al., 2014; Tůmová et al., 2011), y se ha determinado también una mayor fuerza en la cáscara de huevos de gallinas de pastoreo en comparación con los de gallinas de jaulas convencionales, característica que atribuyeron al mayor acceso y consumo de calcio de estas gallinas, el cual obtienen de la tierra (Arbona, 2011; Perić et al., 2016). 
A pesar de que los resultados obtenidos por otros investigadores no pueden ser extrapolados a nuestro país, debido a las diferencias de clima, ambiente, manejo, razas, líneas genéticas y edades de las gallinas, alimentación y otros factores, estos permiten complementar el estudio realizado. Los resultados obtenidos en la prueba de sabor, concordaron con lo que otros autores han reportado. Las diferencias en la alimentación de las gallinas pueden influir en el sabor de los huevos (Kosmidou et al., 2009).

Aunque en este estudio se logró concluir que hubo diferencias de sabor entre los dos tipos de huevo, ya existía un estudio realizado en las comunidades de Costa Rica que determinó que los costarricenses buscan el huevo entre muchas razones, por su buen sabor. A la hora de escoger entre huevos de gallinas de jaulas convencionales y huevos de gallinas de pastoreo, prefieren el huevo de pastoreo, ya que consideran que tiene un mejor sabor (Peña et al., 2011).

A nivel mundial existen otros resultados que indican que los huevos de producción convencional tienen mejores atributos de aroma, sabor y textura (Tercic et al., 2012), mientras que otros autores encontraron que no hubo diferencias sensoriales en los huevos que provienen del sistema de producción convencional en comparación con sistemas de producción alternativos (Mizumoto et al., 2008).

\section{Conclusiones}

En general, los recuentos microbiológicos de los huevos producidos bajo el sistema de pastoreo fueron más altos que los encontrados en huevos producidos bajo el sistema convencional. Esto puede tener relación y explicarse con base en el hallazgo, también experimental, de que los recuentos microbiológicos de muestras de superficies fueron significativamente mayores en el sistema de producción de pastoreo que en el convencional.

El color de yema fue el único parámetro fisicoquímico en el que se encontraron diferencias significativas entre los huevos de producción convencional y los de pastoreo.

Los huevos de producción convencional y de pastoreo analizados presentaron diferencias en su sabor.

Los resultados obtenidos en esta investigación permitieron obtener una perspectiva de las condiciones actuales de producción y manejo de huevos de pastoreo, de la cual se tiene muy poca información a nivel nacional. Permite, además, contrastar este sistema alternativo con el sistema de jaulas convencionales, el cual es el de mayor volumen de producción. La información recopilada puede llegar a ser valiosa tanto para los productores nacionales como para los consumidores. Los productores pueden mejorar el manejo y manipulación de los huevos y así brindarle un producto de mejor calidad a los consumidores nacionales.

\section{Referencias}

Arbona, D. V. (2011). A comparative analysis of freerange vs caged layer hens: egg productivity and quality, humoral immunocompetency \& follicular expression of glucocorticoid receptors [Master's Thesis, North Carolina State University], North California State University libraries. http://www.lib.ncsu.edu/resolver/1840.16/6955

Association of Official Agricultural Chemists. (2005a). AOAC official method 981.12 pH of acidified foods. http://www.eoma. aoac.org/methods/info.asp?ID=18372

Association of Official Agricultural Chemists. (2005b). AOAC official method of analysis 991, 14 coliform and Escherichia coli counts in foods. http://www.eoma.aoac.org/methods/info.asp?ID=46949

Bovšková, H., Míková, K., \&, Panovská Z. (2014). Evaluation of egg yolk color. Czech Journal Of Food Science, 32, $213-217$. https://doi.org/10.17221/47/2013-CJFS

Agron. Mesoam. 33(1): Artículo 46140, 2022 ISSN 2215-3608 doi:10.15517/am.v33i1.46140 
Bray, H. J., \& Ankeny, R.A. (2017) Happy chickens lay tastier eggs: motivations for buying free-range eggs in Australia. Anthrozoös, 30(2), 213-226. https://doi.org/10.1080/08927936.2017.1310986

Caner, C., \& Yuceer, M. (2015). Efficacy of various protein-based coating on enhancing the shelf life of fresh eggs during storage. Poultry Science, 94, 1665-1677. https://doi.org/10.3382/ps/pev102

Centers for Disease Control and Prevention. (2011). National enteric disease surveillance: Salmonella surveillance overview. http://www.cdc.gov/nationalsurveillance/PDFs/NationalSalmSurveillOverview_508/

Central America Data. (2014). Crece la demanda de huevos en Costa Rica. http://www.centralamericadata.com/es/article/home

Clerici, F., Casiraghi, E., Hidalgo, A., \& Rossi, M. (2006, September 10-14). Evaluation of eggshell quality characteristics in relation to the housing system of laying hens [Conference presentation]. European Poultry Conference 2006, Verona, Italy.

Coutts, J. A., \& Wilson, G. C. (Eds.). (2007). Optimum egg quality: a practical approach. 5M Publishing.

De Reu, K. (2006). Bacteriological contamination and infection of shell eggs in the production chain [Doctoral dissertation, University of Ghent]. CORE open. https://core.ac.uk/download/pdf/55719222.pdf

De Reu, K., Messens, W., Heyndrlckx, M., Rodenburg, B., Uyttendaele, M., \& Herman, L. (2008). Bacterial contamination of hen's table eggs and its influencing by housing systems. World's Poultry Science Journal, 64(1), 5-19. https://doi. org/10.1017/S0043933907001687

De Reu, K., Rodenburg, T. B., Grijspeerdt, K., Messens, W., Heyndrickx, M., Tuyttens, F. A. M., Sonck, B., Zoons, J., \& Herman, L. (2009). Bacteriological contamination, dirt, and cracks of eggshells in furnished cages and noncage systems for laying hens: An international on-farm comparison. Poultry Science, 88, 2442-2448. https://doi.org/10.3382/ps.2009-00097

Eke, M. O., Olaitan, N. I., \& Ochefu, J. H. (2013). Effect of storage conditions on the quality attributes of shell table eggs. Nigerian Food Journal, 31(2), 18-24. https://doi.org/10.1016/S0189-7241(15)30072-2

Englmaierová, M., Tumova, E., Charvatova, V., \& Skrivan, M. (2014). Effects of laying hens housing system on laying performance, egg quality characteristics and egg microbial contamination. Czech Journal of Animal Science, 59(8), 345-352. https://doi.org/10.17221/7585-CJAS

Ennis, J. M., \& Jesionka, V. (2011). The power of sensory discrimination methods revisited. Journal of Sensory Studies, 26, 371-382. https://doi.org/10.1111/j.1745-459X.2011.00353.x

Fischer, J. (2010). Influence of housing system on bacterial eggshell contamination and horizontal transmission of Salmonella and Campylobacter among laying hens [Doctoral dissertation, University of Georgia]. University of Georgia Libraries. https://getd.libs.uga.edu/pdfs/hannah_jackie_f_201012_phd.pdf

Food and Drug Administration. (2018). Bacteriological Analytical Manual. https://www.fda.gov/food/laboratory-methods-food/ bacteriological-analytical-manual-bam

Food Standards Australia New Zealand. (2016). Compendium of Microbiological Criteria for Food. Food Standards Australia New Zealand Publishing. https://www.foodstandards.gov.au/publications/Documents/Compedium\%20 of\%20Microbiological\%20Criteria/Compendium_revised-Sep\%202018.pdf

Fronding, G. W. (1998, June 2-3). Egg processing: ideas for the future [Conference Presentation]. Egg Processing Workshop, California, United States. https://www.yumpu.com/en/document/read/19008238/egg-processing-ideas-for-the-futureglenn-froning

Agron. Mesoam. 33(1): Artículo 46140, 2022

ISSN 2215-3608 doi:10.15517/am.v33i1.46140 
Giacomozzi, J. (2014). Situación actual de la industria del huevo. Oficina de estudios y políticas agrarias de Chile. https://www. odepa.gob.cl/publicaciones/articulos/situacion-actual-de-la-industria-del-huevo-junio-de-2014

Gjorgovska, N., Filev, K., \& Levov, V. (2011). External and internal quality of eggs produced from aged hens. Lucrari Stiintifice, $56,342-345$.

Greco, M. V., Franchi, M. L., Rico S. L., Pardo, A. G., \& Pose, G. N. (2014). Mycotoxins and mycotoxigenic fungi in poultry feed for food-producing animals. The Scientific World Journal, 2014, Article 968215. https://doi.org/10.1155/2014/968215

Hannah, J. F., Wilson, J. L., Cox, N. A., Cason, J. A., Bourassa, D. V., Musgrove, M. T., Richardson, L. J., Rigsby, L. L., \& Buhr, R. J. (2011). Comparison of shell bacteria from unwashed and washed table eggs harvested from caged laying hens and cage-free floor-housed laying hens. Poultry Science, 90, 1586-1593. https://doi.org/10.3382/ps.2010-01115

Holt, P. S., Davies, R. H., Dewulf, J., Gast, R. K., Huwe, J. K., Jones, D. R., Waltman D., \& Willian, K. R. (2011). The impact of different housing systems on egg safety and quality. Poultry Science, 90, 251-262. https://doi.org/10.3382/ps.2010-00794

Hui, Y. H. (2014). Plant sanitation for food processing and food service (2 ${ }^{\text {nd }}$ Ed.). CRC Press.

Jaramillo, A. H., Mogica, J., Caro, E. A., \& Sosa, J. (2018). Evaluación de la calidad del huevo de gallina en dos sistemas de alojamiento - piso convencional con suplementación de sauco (Sambucus nigra) y pastoreo con kikuyo (Pennisetum clandestinum)- en la Sabana de Bogotá. Revista Siembra CBA, 2(1), 59-77. http://revistas.sena.edu.co/index.php/ Revsiembracba/article/view/1881/1987

Jones, D. R., \& Anderson, K. E. (2013). Housing system and laying hen strain impacts on egg microbiology. Poultry Science, 92, 2221-2225. https://doi.org/10.3382/ps.2012-02799

Jones, D. R., Anderson, K. E., \& Guard, J. Y. (2012). Prevalence of coliforms, Salmonella, Listeria and Campylobacter associated with eggs and the environment of conventional cage and free-range egg production. Poultry Science, 91, 1195-1202. https://doi.org/10.3382/ps.2011-01795

Jones, D. R., Anderson, K. E., \& Musgrove, M. T. (2011). Comparison of environmental and egg microbiology associated with conventional and free-range laying hen management. Poultry Science, 90, 2063-2068. https://doi.org/10.3382/ ps.2010- 01139

Jones, D. R., Cox, N. A., Guard, J., Fedorka, P. J., Buhr, R. J., Gast, R. K., Abdo, Z., Rigsby, L. L., Plumblee, J. R., Karcher, D. M., Robinson, C. I., Blatchford, R. A., \& Makagon, M. M. (2015). Microbiological impact of three commercial laying hen housing systems. Poultry Science, 94, 544-551. https://doi.org/10.3382/ps/peu010

Jones, D. R., Karcher, D. M., \& Abdo, Z. (2014). Effect of a commercial housing system on egg quality during extended storage. Poultry Science, 93, 1282-1288. https://doi.org/10.3382/ps.2013-03631

Karcher, D. M., Jones, D. R., Abdo, Z., Zhao, Y., Shepherd, T. A., \& Xin, H. (2015). Impact of commercial housing systems and nutrient and energy intake on laying hen performance and egg quality parameters. Poultry Science, 94, 485-501. https://doi.org/10.3382/ps/peu078

Kenji, J. (2010, August 27). The Food Lab: do better eggs really taste better? Serious Eats. http://www.seriouseats.com/2010/08/ what-are-the-best-eggs-cage-free-organic-omega-3s-grocery-store-brand-the-food-lab.html

Ketta, M., \& Tůmová, E. (2016). Eggshell structure, measurements, and quality-affecting factors in laying hens: A review. Czech Journal of Animal Science, 61(7), 299-309. https://doi.org/10.17221/46/2015-CJAS

Agron. Mesoam. 33(1): Artículo 46140, 2022 ISSN 2215-3608 doi:10.15517/am.v33i1.46140 
Kosmidou, M., Sossidou, E., Fortomaris, P., Yannakopoulos, A., \& Tserveni-Goussi, A. (2009, June 23-26). Sensory evaluation of eggs produced by free-range hens foraging in pastures enriched with Ocimum basilicum and Mentha spicata [Conference Presentation]. European Symposium on the Quality of Eggs and Egg Products, Prague, Czech Republic. https://www.cabi.org/Uploads/animal-science/worlds-poultry-science-association/WPSA-turkey-2009/19_ kosmidou_mps2009.pdf

Mizumoto, E. M., Canniatti-Brazaca, S. G., \& Machado, F. M. (2008). Chemical and sensorial evaluation of eggs obtained by different production systems. Food Science and Technology, 28(1), 60-65. https://doi.org/10.1590/S010120612008000100010.

Moats, W. A. (1980). Classification of bacteria from commercial egg washers and washed and unwashed eggs. Applied and Environmental Microbiology, 40(4), 710-714.

Mugnai, C., Del Bosco, A., \& Castellini, C. (2009). Effect of rearing system and season on the performance and egg characteristics of Ancona laying hens. Italian Journal of Animal Science, 8(2), 175-188. https://doi.org/10.4081/ ijas.2009.175

Musgrove, M. T., Jones, D. R., Shaw, J. D., Sheppard, M., \& Harrison, M. A. (2009). Enterobacteriaceae and related organisms isolated from nest run cart shelves in commercial shell egg processing facilities. Poultry Science, 88, 2113-2117. https://doi.org/10.3382/ps.2009-00021

Parisi, M. A., Northcutt, J. K., Smith, D. P., Steinberg, E. L., \& Dawson, P. L. (2015). Microbiological contamination of shell eggs produced in conventional and free-range housing systems. Food Control, 47, 161-165. https://doi.org/10.1016/j. foodcont.2014.06.038

Peña, M., Castro, A. C., \& Martínez, T. (2011). Conocimientos, opiniones y prácticas respecto al huevo de gallina en familias de comunidades urbana-rural, Costa Rica. Revista Costarricense de Salud Pública, 20(1), 32-39.

Perić, L., Dukic, M., \& Bjedov, S. (2016). Effect of production systems on quality and chemical composition of table eggs. The Serbian Journal of Agricultural Sciences, 65(3), 27-31. https://doi.org/10.1515/contagri-2016-0014

Poder Ejecutivo. (2006). Decreto $N^{\circ} 33115$ de 2006. Reglamento técnico de Costa Rica 397:2006: Huevos frescos o refrigerados de gallina para consumo humano. Ministerio de Agricultura y Ganadería. http://www.mag.go.cr/legislacion/2006/ de-33115.pdf

Poder Ejecutivo. (2018). Decreto $N^{\circ} 41420$ de 2018: Reglamento técnico centroamericano RTCA 67.04.50:17: Alimentos. Criterios microbiológicos para la inocuidad de los alimentos. Ministerio de Comercio Exterior. https://www.comex. go.cr/media/3480/279_anex-243-rtca-67-04-50-08-microbio-may-09.pdf

Pouch, F., \& Ito, K. (Eds.) (2001). Compendium of methods for the microbiological examination of foods. American Public Health Association.

Protais, J., Queguiner, S., Boscher, E., Piquet, J. C., Nagard, B., \& Salvat, G. (2003, September 23-26). Effect of housing system on the bacterial flora in the air and on egg shells [Conference Presentation]. European Symposium of the Quality of Eggs and Egg Products. France. http://www.wpsa.com/index.php/publications/wpsa-proceedings/effect-of-housingsystems-on-the-bacterial-flora-in-the-air-and-on-egg-shells/viewdocument/3494

Ruiz, B. (2020). Fuerte crecimiento de la avicultura latinoamericana en 2019. Industria Avícola, 67(3), 4-25.

Sharaf Eddin, A., Ibrahim, S., \& Tahergorabi, R. (2019). Egg quality and safety with an overview of edible coating application for egg preservation. Food Chemistry, 296, 29-39. https://doi.org/10.1016/j.foodchem.2019.05.182 
Singh, R., Cheng, K. M., \& Silversides, F. G. (2009). Production performance and egg quality of four strains of laying hens kept in conventional cages and floor pens. Poultry Science, 88, 256-264. https://doi.org/10.3382/ps.2008-00237

Smeltzer, T. I., Orange, K., Peel, B., \& Runge, G. I. (1979). Bacterial penetration in floor and nest box eggs from meat and layer birds. Australian Veterinary Journal, 55, 592-593. https://doi.org/10.1111/j.1751-0813.1979.tb07055.x

Smith, A., Rose, S. P., Wells, R. G., \& Pirgoziieb, V. (2000). The effect of changing the excreta moisture of caged laying hens on the excreta and microbial contamination of their egg shells. Poultry Science, 41, 168-173. https://doi. org/10.1080/713654903

Stojanova, M., Kocevski, D., Vukovic, V., \& Porcu, K. (2016). Comparison of qualitative properties of eggs from hens reared in battery cages and free range system. International Journal of Agronomy and Agricultural Research, 9(6), 77-83. https://www.innspub.net/wp-content/uploads/2016/12/IJAAR-V9No6-p77-83.pdf

Sulimova, L., Zhuchaev, K., \& Kochneva, M. (2020). Poultry behavior reactions and welfare. Sel'skokhozyaistvennaya Biologiya, 55(2), 209-224. http://www.agrobiology.ru/2-2020sulimova-eng.html

Svobodová, J., \& Tůmová, E. (2014). Factors affecting microbial contamination of market eggs: a review. Scientia Agriculturae Bohemica, 45(4), 226-237. https://doi.org/10.1515/sab-2015-0003

Tercic, D., Zlender, B., \& Holcman, A. (2012). External, internal and sensory qualities of table eggs as influenced by two different production systems. Agro-knowledge Journal, 13(4), 555-562. https://doi.org/10.7251/AGREN1204555T

Tomczyk, Ł., Stępień, Ł., Urbaniak, M., Szablewski, T., Cegielska-Radziejewska, R., \& Stuper-Szablewska, K. (2018). Characterisation of the mycobiota on the shell surface of table eggs acquired from different egg-laying hen breeding systems. Toxins, 10(7), Article 293. https://doi.org/10.3390/toxins 10070293

Tůmová, E., \& Ebeid, T. (2003). Effect of housing system on performance and egg quality characteristics in laying hens. Scientia Agriculturae Bohemica, 34, 73-80.

Tůmová, E., Englmaierová, M., Ledvinka, Z., \& Charvatova, V. (2011). Interaction between housing system and genotype in relation to internal and external egg quality parameters. Czech Journal of Animal Science, 56(11), 490-498. https:// doi.org/10.17221/3838-CJAS

Yenice, G., Kaynar, O., Ileriturk, M., Hira, F., \& Hayirli, A. (2016). Quality of eggs in different production systems. Czech Journal of Food Science, 31(4), 370-376. https://doi.org/10.17221/33/2016-CJFS

Wilson, A., Chandry, S., Turner, M. S., Courtice, J. M., \& Fegan, N. (2021). Comparison between cage and free-range egg production on microbial composition, diversity and the presence of Salmonella enterica. Food Microbiology, 10, Article 103754. https://doi.org/10.1016/j.fm.2021.103754 